\title{
Les possibilités d'utilisation ciblée des ressources de la Responsabilité Sociale des Entreprises dans le secteur vinier dans le but d'améliorer la durabilité et la spécificité culturelle des régions viticoles / Opportunities of purposeful use of corporative social responsibility resources in the Wine Sector, to enhance the stability and cultural uniqueness of winery regions
}

\author{
Ivana Murdjeva \\ Sofia City Province, Sofia, Bulgaria
}

\begin{abstract}
Résumé. Ces dernières années, la responsabilité corporative sociale (RCS) marque une certaine croissance dans l'intérêt des entreprises envers l'intégration dans des causes d'intérêt social dans leurs pratiques commerciales. L'on note un intérêt accru envers les sujets liés à l'éducation, l'écologie, la santé, l'amélioration de la sensibilisation des consommateurs, l'amélioration des conditions de travail et, tout dernièrement, aux sujets et aux initiatives visant la conservation et la protection du patrimoine culturel matériel et immatériel. Diverses entreprises et produc-teurs, dans leur plupart de petite et moyenne envergure, dirigent leurs ressources vers un tra-vail avec des groupes et des communautés locales, afin d'améliorer le milieu et de créer une motivation pour la conservation durable des emplois et l'augmentation de l'engagement pro-fessionnel et des compétences des personnes employées dans la production. Les secteurs publics ayant contact avec la culture, l'art, la préservation de l'identité et des tra-ditions manquent encore de popularité, y compris les productions spécifiques aux régions, la préservation du milieu et la conservation des phénomènes de la nature, la préservation de la culture des communautés locales et leur intégration dans un échange d'ordre global.
\end{abstract}

\begin{abstract}
Corporative Social Responsibility (CSR) has marked a growth with respect to the interest of companies to integrate some socially important causes into their business practices during last years. A strengthened interest to some topics related to education, ecology, health, improvement of users' information capacity, improvement of labor conditions and, ultimately, also to topics and incentives related to safe-keeping and protection of cultural material and intangible patrimony is noticed. Single companies and manufacturers, small and median mainly, are orienting their resources to the collaboration with local societies and groups, in order to improve the environment and to create a motivation of durable preservation of the number or places of work and enhancement of professional commitments and competence of the persons employed in the manufacturing process. Public Sectors relating to culture, arts, preservation of identity and traditions are still less popular, including, but not limited to specific regional productions, landscape and natural phenomena preservation, conservation of the culture of local communities and its integration into an overall exchange.
\end{abstract}

\section{Analyse de la situation}

La base de cette analyse repose sur des observations de longue date de l'état et du développement des régions viticoles qui, en ce moment, disposent d'une structure insuffisamment développée, une faible stabilité du marché et une faible compétitivité. L'attention envers ces régions est provoquée par leur dynamique peu typique de développement, et elles représentent un terrain d'intérêt d'analyse des possibilités d'utilisation des ressources de la Responsabilité sociale des entreprises (RSE), afin d'augmenter la stabilité, le développement des régions et le rétablissement des régions et leur réputation de marché.
Pour en arriver à ses conclusions, cette étude a eu recours à des observations sur la structure et le développement des régions viticoles en Bulgarie, car nous considérons que les résultats peuvent être appliqués avec succès sur d'autres domaines, correspondant aux caractéristiques de base au moment de l'étude :

- L'éloignement

- Une faible structure interne

- Une partie insignifiante de marché

- Une faible compétitivité

- Une perte de réputation

- Un faible intérêt des fabricants et une faible identification et la confiance de la part des consommateurs. 
L'actualité de la recherche est soutenue par la tendance établie au cours des dernières années d'accroissement d'intérêt du marché envers les régions viticoles plus ou moins connus, envers la conservation et la promotion des cépages locaux et la manutention des traditions spécifiques et des méthodes de production de vin. Il va sans dire que dans la définition de la portée de l'étude il y eut des débats de plus en plus soutenus concernant le soutien et la préservation des industries locales dans un marché global à travers le développement et la mise à jour du système de protection d'origine.

Le territoire de la Bulgarie est conditionnellement divisé en cinq régions viticoles, chacune avec un sol spécifique et une structure climatique - la vallée de la Thrace, la vallée du Danube, la vallée de la rivière Strouma, la région de la mer Noire et la région de la Vallée des Roses. À l'heure actuelle, la plaine de Thrace et la vallée du Danube existent comme des indications géographiques protégées, la vallée de la rivière Strouma comme une appellation d'origine, la région de la mer Noire, divisée en deux appellations d'origine : le sud-ouest et la côte de la mer du Nord.

La région de la Vallée des Roses se trouve hors du système de protection et d'utilisation pratique dans l'étiquetage des vins. Sur la base de cette division en zones, Bulgarie a identifié et protégé encore 51 appellations d'origine, inégalement éparses sur l'ensemble du territoire. La plupart de ces nom ont connu leur apogée de développement lors et autour des 80 -s du XX siècle, sous les conditions complètement différentes nationale et de conjoncture mondiale et à l'heure qu'il est ils se trouvent dans la phase inférieure de leur développement, couvrant des parts négligeables du marché. Seules les zones avec une indication géographique protégée - la Plaine de Thrace et la Vallée du Danube enregistrent un développement et une augmentation de leur part de marché.

Chacune des régions décrites montre une structure socio-économique différente, une distribution de l'exploitations de viticulture et de la structure des producteurs de vin du point de vue de leur volume, résilience, ressources, marché ciblé et priorités du point de vue de la RSE. Les zones ont une structure interne faible et un manque de structures organisationnelles qui puisse entreprendre un contrôle professionnel et une protection de l'appellation. En raison de la variété des entités dans la zone (différence dans la structure de production, capacité, durabilité, ciblage de marché, nomenclature des produits), la structure est très difficile à établir rassemblant des éléments qui puissent servir de base pour une stratégie commune pour la mise en œuvre des mécanismes de la RSE, la protection et le développement de la région.

Il y a une absence de conditions de base pour faciliter l'intégration des principes de la RSE dans la structure de commercialisation des entreprises, telles que : présence de groupes ou réseaux de producteurs qui puissent mener des activités de décentralisation ou délocation, réduction des coûts de production et de distribution, etc. qui soient introduits dans les entreprises de gestion unifiée et une uniformité de standardisation des produits. Une partie importante des entreprises indique une faible connaissance des mécanismes de la RSE et une non/application de ces mécanismes dans leurs stratégies de marché. Dans ces cas de différences substantielles des sujets dans une région et compte tenu de nouvelles approches de la RSE en tant qu'une approche organisationnelle au sein de l'entreprise, autour de laquelle tous les intervenants sont coordonnés afin d'atteindre leurs objectifs communs, la réalisation de la stratégie territoriale pour la RSE fait face à des difficultés importantes telles que :

- Une structure organisationnelle différente des entreprises

- Une différence de volume de production, de stabilité et d'emploi dans les entreprises

- Une différence dans l'approche de marketing

- Une différence d'approche dans la région (présence de producteurs qui n'ont pas de rapport envers la zone, développant un portefeuille en fonction des marques d'origine unique).

Dans un plan positif, l'on devrait souligner que malgré le manque de connaissances des principes de la RSE, ces dernières années la responsabilité sociale marque une croissance dans l'intérêt des entreprises. Elles sont plus en plus informées des causes socialement significatives et appliquent, bien que partiellement, des mécanismes pour l'intégration de leurs pratiques commerciales. L'on note l'intérêt dans les sujets liés à l'éducation, l'écologie, la santé, l'amélioration de la sensibilisation des consommateurs, l'amélioration des conditions de travail. L'intérêt envers les sujets et les initiatives relatives au stockage et à la protection du patrimoine culturel matériel et immatériel est toutefois plus faible. Diverses entreprises et fabricants, notamment les petites et moyennes, dirigent leurs ressources dans le domaine du travail avec les communautés et groupes locaux, afin d'améliorer l'environnement et de créer une motivation pour la conservation permanente des emplois et l'augmentation de l'engagement professionnel et les compétences des personnes engagées dans la production.

Il va sans dire, si au début de l'activité des sociétés l'on pourrait envisager une certaine « fatigue » de l'idée. L'on enregistre la présence de davantage de suggestions pour engager les entreprises dans les causes publiques, parmi elles l'on trouve souvent certaines ayant un caractère imitatif copiant des idées réussies, sans aucune garantie de stabilité et d'utilité du résultat final. Les entreprises courent un risque de s'orienter de façon difficile et de trouver des idées originales, il est de plus en plus difficile de faire confiance aux nouvelles formations et de s'engager dans des causes non vérifiées. C'est alors qu'apparait le risque que certains secteurs publics restent définitivement hors de leur portée d'intérêt en raison de l'activité plus faible ou plus difficile pour les formations.

Les secteurs qui sont liées à la culture, l'art, la préservation de l'identité et les traditions, y compris les industries spécifiques dans les régions, la préservation du paysage et la conservation des phénomènes naturels, la préservation de la culture des communautés locales et de l'intégration dans un inter change général sont toujours peu populaires.

\section{Stratégie territoriale pour la mise en œuvre des mécanismes de la RSE - avantages et défis}

Les producteurs de vin, laissant de côté les plus grandes structures d'entreprise, dans un certain sens, se trouvent 
dans un processus d'activation de l'intérêt envers la RSE, en tant que le cadre d'une stratégie de marketing et un facteur de l'augmentation des compétitivités individuelles. Les raisons s'en trouve dans toute une série de facteurs, mais surtout dans la spécificité du produit - le vin, formant l'identité de la base d'origine, de tradition et la réputation de la région et du producteur. Nous dirions que les producteurs de vin ont une position privilégiée pour surveiller le processus de mise en œuvre des mécanismes de la RSE et de comparer les résultats avec une certaine vigilance, même s'ils ne tombent pas sous la « fatigue » de l'idée.

Comme règle générale, les pratiques commerciales chez les fabricants de vins sont liées à la zone de production. Ils prennent en compte la corrélation directe entre la qualité du produit et le développement de la région - publique, infrastructures, éducation, santé, préservation des communautés autochtones, de la culture et de l'art. Chaque cause abstraite et séparée du territoire de production n'aurait pas de sens pour les producteurs de vin, du point de vue du champ d'application de la RSE en tant que stratégie intégrée dans la gestion de l'entreprise. Les principaux éléments de la RSE : marketing, lié à une cause, promotion d'une cause, entreprise de marketing social, le bénévolat et la philanthropie ne fonctionnerait pas ou auraient un moindre effet s'ils ne sont pas activement et spécifiquement ciblés sur la zone de production. La spécificité de l'engagement avec la zone de production rend le producteurs de vin un conducteur naturel des causes publiques, liées à la région et même plus encore avec la préservation du patrimoine culturel, de l'art et de culture, la préservation des caractéristiques spécifiques des régions, la préservation du paysage et de ses pratiques agro-écologiques associées. En ce sens, les producteurs de vin ont été précédemment visés en ce qui concerne les causes qui soutiennent ou soutiendraient la zone.

Des études récentes dans le domaine de la RSE et l'efficacité de la mise en œuvre de ses mécanismes, en vue de l'efficacité marketing de l'entreprise, indiquent qu'il est plus approprié que les éléments de la RSE soient utilisés de façon intégrée avec les bonnes pratiques de fabrication, adoptés par le fabricant. En règle générale, les bonnes pratiques de fabrication appliqués par les producteurs de vin, sont directement ou indirectement liées aux spécificités de la région, formant la qualité du produit (caractéristiques climatiques et du sol, pratiques traditionnelles appliquées pour produire du vin de cépages et pour la vinification, la tradition culinaire locale, les coutumes, la spécificité des produits agricoles cultivés dans la région, etc.). Cette pré condition fait une fois de plus de sorte que les producteurs de vin sont conduits dans un groupe cible d'acteurs liée en permanence à la zone de production.

Il y a aussi un fait incontestable : que l'application de stratégies territoriales pour la RSE est plus efficace que l'application des mécanismes éloignés des objectifs directs des entreprises. Les stratégies territoriales ont l'avantage de prendre en compte les besoins spécifiques d'un groupe d'entités référencées dans une région donnée et d'offrir des solutions et des objectifs autour desquels la réalisation d'une unification serait possible. La stratégie territoriale offre davantage de possibilités de ralliement aux bonnes pratiques de fabrication adoptées par les producteurs dans les régions. Et encore, la stratégie territoriale pour la RSE est une base appropriée pour la formation de réseaux ou de groupes d'entreprises appliquant une gestion uniforme et/ou des normes de qualité.

En ce qui concerne les producteurs de vin opérant dans des zones viticoles périphériques, avec un petit marché d'action territoriale, la stratégie de la RSE aurait été une base appropriée pour l'édification d'une structure interne efficace de la région et la récupération de son importance sur le marché et de son importance comme un facteur majeur dans la formation de la valeur ajoutée du produit. En ce sens, par le biais de la perception de la stratégie territoriale de la RSE au profit de la région et des entités y opérant ces derniers auraient trouvé la solution aux problèmes suivants :

- Renforcement du caractère pragmatique de l'interaction entre les particularités et les besoins de la région et la pratique et les objectifs économiques des producteurs.

- Surmonter le manque de motivation chez les producteurs d'investir dans des stratégies de commercialisation liées à la région, sous condition que cette dernière est en dehors du chiffre d'affaires du commerce et/ou ayant un faible marketing.

- Création d'une base solide pour construire ou restaurer l'infrastructure interne de la région.

- Démarrage du processus de rétablissement de la réputation du marché et de l'identité de la région.

- Création des conditions pour l'unification des modèles organisationnels et des bonnes pratiques de fabrication chez les producteurs - condition nécessaire sine qua non pour la mise en œuvre et l'application de normes communes régionales pour la qualité du produit.

En bref, la stratégie territoriale pour la RSE répond de façon efficace aux questions suivantes: ce que nous pouvons et ce que nous voulons faire ensemble dans la région où nous opérons, afin qu'elle nous assure une distinction et un secteur de marché correspondant aux objectifs individuels de chacun des acteurs ? Quelles sont les ressources de la région, que nous pouvons utiliser avec un consentement mutuel, afin qu'ils puissent être utilisés, stockés et mis au point? Quel type d'investissements dans des activités non économiques pourrait nous proposer la région, et ont-ils le potentiel réel pour en fait reconstruire l'espace de façon convenable pour la communication de marketing ?

Il est d'une importance cruciale pour le succès de la stratégie territoriale de la RSE d'y avoir, à part les exigences spécifiques de la région, d'intégrer tout aussi les intérêts des sujets, permettant la réalisation de l'accord, bien entendu, sous forme de réduction pour atteindre l'accord général. La stratégie territoriale dans laquelle les entreprises de la région ne reconnaissent pas leurs objectifs spécifiques n'aurait été ni efficace, ni réussie.

En revanche, les opposants de la stratégie territoriale pour la RSE, auront indiqué que l'objectif principal de la stratégie - rétablir la réputation de la région grâce à un contrôle commun sur l'origine et la qualité du produit obtenu dans ses limites, pourrait être résolu avec davantage de succès par l'adoption de législations régionales sectorielles.

L'état actuel des structures législatives se tournent de plus en plus rarement vers la législation sectorielle régionale, adoptant une norme de validité universelle correspondant mieux à la société moderne générale 
(droit communautaire). Même les lois régissant la production des produits sous la protection de l'origine (des indications géographiques et des appellations d'origine) ne peuvent être définies comme des lois régionales. Leur vérification doit être effectuée à niveau national, avec l'introduction du système E-Bacchus ainsi qu'au niveau communautaire. Les régions, par l'intermédiaire de ses autorités administratives ne saurait invoquer des dérogations à la règle générale en faveur de la spécificité locale au micro-niveau. Une approche similaire pourrait être destructive pour la norme commune réglementant le marché commun et permettant par exception une déviation dans les cas de partialité explicitement déclarés. En ce sens, la législation sectorielle régionale n'est pas la réponse cherchée et ne peut déplacer ou rendre nulle une stratégie territoriale pour la RSE.

Certainement, la législation régionale peut créer les conditions qui encouragent les producteurs à appliquer les mécanismes de la RSE, en étant conformes à la nature volontaire de responsabilité sociale. Il serait important, en ce sens, d'avoir eu des dispositions liées à la philanthropie, au mécénat, aux partenariats publics-privés, etc.

\section{Rétablissement de l'importance économique de la région. Édification d'une image positive de la zone. Rôle de la stratégie territoriale pour la RSE dans le processus}

Comme mentionné dans cette étude, la même est dirigée vers les régions vitivinicoles périphériques ayant perdu leur part de marché et la réputation commerciale. Il fut indiqué que ces zones sont intéressantes à étudier car elles montrent une dynamique de développement atypique pour les régions viticoles. Dans la plupart des cas, les régions observées ont passé leur pic de développement, n'ont pas retenu leur part de marché et ont cessé d'être économiquement efficaces. Les raisons pourraient être disposées dans un large éventail de relations socioéconomique, et que dans le cas examiné, elles pourraient se concentrer dans un changement radical des relations entre le marché et système politique qui les gère. Les régions en Bulgarie ont eu leur pic de développement dans les années 1970 et 1980 du XXème siècle, leur production ayant suivi les règles introduites dans un système de marché qui diffère complètement du marché libéral. Après la réforme politique de la fin du siècle passé, la structure organisationnelle des producteurs de vin est radicalement changée. Les régions sont soumises au défi d'un marché déjà établi, dont ils ont la souplesse nécessaire et la possibilité d'adaptation au marketing. Ainsi, ils perdent leur valeur marchande et leur réputation. La motivation des producteurs pour produire dans la région, dans les limites liées à la préservation et la protection de l'origine et le nom, est perdue.

Le rétablissement de la valeur économique de la région et la construction d'une image positive à ce point n'est plus une restauration de l'image de la région du temps de son apogée. Les producteurs sont placés devant la tâche de parvenir à un accord sur la construction d'une nouvelle image de marketing de la région et de promouvoir sa nouvelle identité. Souvent, la situation socio-économique dans les régions est telle qu'il faut une nouvelle édification de la communauté locale, qui aura une attitude motivée pour préserver la spécificité du territoire. La restauration de l'importance économique de la région doit passer par les étapes suivantes :

1. Identification et développement de la communauté locale.

2. Identification des ressources non économiques de la zone.

3. Identification des besoins de la région du point de vue de la communauté locale.

4. Construction d'une infrastructure organisationnelle interne de la région.

5. Analyse des approches possibles de marché pour la zone. Choix d'une stratégie de marketing appropriée et commune.

6. Identification et acceptation du catalogue de produits, représentant au degré complet la toute nouvelle apparence du marché de la zone.

L'identification des ressources non économiques de la région et leur lien avec les besoins immédiats de la communauté locale, représente la partie la plus intéressante et complexe de la restauration de l'importance du marché de la région et l'imposition de sa nouvelle image.

Il est nécessaire de faire la précision suivante. L'étude se concentre sur les domaines traditionnels de la production de vin, c'est-à-dire les zones ayant une histoire, décrite sur la base de la spécificité du sol et du climat et de la distinction du produit. La perte de leur valeur marchande est en raison de l'appauvrissement ou la perte des ressources de la région et, en résultat de circonstances externes à la zone. En ce sens, nous ne discuterons pas la mise en place d'une zone « nouvelle $»$ au moyen de méthodes de marketing, sinon un «avivement » d'une région traditionnelle existante. Cette précision est importante du point de vue de la possible utilisation spéculative des conclusions susmentionnées concernant de nouvelles zones pour lesquelles les approches devraient être complètement différentes.

L'identification des ressources non économiques d'une région définie est une tâche qui pourrait prendre un temps considérable si elle est mal positionnée dans la relation des activités en séquence. Pratiquement, elle devrait être une des premières tâches posées ensemble ou immédiatement après l'identification de la communauté locale, en tant qu'ensemble de caractères sociaux spécifiques et de relations sociales. Il est possible que la communauté locale indique elle-même ou attire l'attention sur des ressources considérées par elle-même comme importantes. L'identification de la portée exacte des ressources non économiques dans la région est une condition préalable pour la création de conditions de base pour surmonter les problèmes énumérés de haute importance pour la communauté locale :

- Structuration organisationnelle de la communauté locale

- Réduction de la migration et du dépeuplement de la région

- Solution des problèmes des emplois à long terme dans le domaine

- Augmentation de la qualification de la main-d'œuvre dans la région 
- Amélioration de l'infrastructure locale dans le domaine de l'éducation et de la culture

- Création d'un engagement personnel envers la zone externe aux personnes étrangères à l'entreprise (les parties intéressées externes).

De leur côté, les ressources non économiques de la zone peuvent se placer dans un très large spectre, ce qui, en plus d'élargir la capacité des producteurs de la région, peut être déterminé en tant que complication principale à leur évaluation correcte et l'établissement des priorités. Les ressources non économiques peuvent être groupées de la façon suivante :

- Education

- Culture de masse

- Possibilités de tourisme alternatif

- Mise de l'accent sur les reliques culturelles et archéologiques et les sites

- Conservation de la tradition culturelle immatérielle

- Richesse locale des variétés spécifiques locales

- Spécificité du paysage, présence de phénomènes naturels uniques présentant de l'intérêt pour la conservation du milieu naturel.

Chacun des groupes pourrait être soumis à un examen individuel. Chacun de ces groupes peut être une base fiable sur laquelle l'on regrouperait les objectifs des sujets de la zone ayant une vision commune pour rétablir sa réputation de marché et sa part de marché. C'est pourquoi chaque groupe peut se trouver à la base d'une stratégie territoriale pour la RSE, en utilisant tout élément: un marketing, lié à une cause, la promotion d'une cause, l'entreprise de marketing social, le bénévolat et la philanthropie.

Il a été constaté que, vers le moment de l'étude, l'intérêt des producteurs envers les différents groupes de ressources non économiques est inégal. L'intérêt accru est dédié au domaine de l'éducation, la protection de l'environnement, l'investissement dans le développement des ressources humaines, la santé, l'amélioration de la sensibilisation des consommateurs, l'amélioration des conditions de travail. L'intérêt, allant de faible à notable, est dédié aux initiatives relatives à la protection du patrimoine culturel, l'éducation liée aux arts et à la culture visuelle.

Les conclusions de cette étude indiquent qu'une stratégie territoriale de la RSE dans le secteur vitivinicole ne serait efficace que si l'on pariait sur des ressources non économiques peu développées, telles que la protection du patrimoine culturel, l'éducation liée aux arts et à culture visuelle, s'il aurait été intégré avec succès parmi les besoins identifiés de la communauté locale.

\section{Infrastructures culturelles dans les régions}

Selon les données des statistiques nationales il y a des 210 musées sur le territoire du pays qui ont enregistré 4763472 visiteurs par an et qui ont un total de 7539761 pièces de musée. 36 , du nombre total des musées du pays, sont des galeries d'art régionales.

En tant qu'institutions culturelles, les musées gèrent une ressource considérable de possibilités, liées en particulier à l'intégration des causes d'importance sociale, organisation d'événements divers en tant qu'hôtes,

\begin{tabular}{|c|c|}
\hline Régions & Musées \\
\hline Au total & 201 \\
\hline Blagoevgrad & 7 \\
\hline Burgas & 12 \\
\hline Varna & 9 \\
\hline Véliko Tarnovo & 10 \\
\hline Vidin & 2 \\
\hline Vratza & 3 \\
\hline Gabrovo & 9 \\
\hline Dobritch & 7 \\
\hline Kardjali & 1 \\
\hline Kyustendil & 5 \\
\hline Lovetch & 6 \\
\hline Montana & 6 \\
\hline Pazardjik & 9 \\
\hline Pernik & 5 \\
\hline Pléven & 5 \\
\hline Plovdiv & 14 \\
\hline Razgrad & 3 \\
\hline Russé & 4 \\
\hline Silistra & 5 \\
\hline Sliven & 5 \\
\hline Smolyan & 3 \\
\hline Sofia & 7 \\
\hline Sofia (capitale) & 27 \\
\hline Stara Zagora & 11 \\
\hline Targovisté & 4 \\
\hline Haskovo & 5 \\
\hline Shumén & 12 \\
\hline Yambol & 5 \\
\hline
\end{tabular}

création et mise en œuvre de différentes activités, des programmes visant à accroître la sensibilisation et l'éducation. La coopération avec les musées et le soutien de l'activité du musée jouissent en général d'un haut degré d'approbation sociale. La tradition nationale perçoit les musées en tant que sites pour l'accumulation de la mémoire nationale et de l'identité. Les musées régionaux conservent la mémoire locale, et les galeries, l’identité visuelle et la tradition de la région.

L'intégration des activités d'appui de structures culturelles locales dans une stratégie territoriale pour la RSE transformerait la stratégie dans un acte de coopération établie non seulement entre les producteurs dans les régions, sinon entre les producteurs et la communauté locale. En pratique, cela signifie la construction ou la reconstruction des infrastructures internes dans la région et le positionnement des producteurs de vin dans la catégorie des entités responsables à échelle régionale, ayant non seulement une attitude portée uniquement sur les bénéfices des sociétés de l'entreprise, mais aussi au développement de la région comme un ensemble de relations publiques dans divers domaines.

La construction d'un nouvel aspect dans ce cas passe par la récupération de la mémoire sur la tradition et la culture dans le domaine de la reconstruction de leur image à travers le prisme des nouvelles relations sociales.

Ayant recours à l'héritage culturel en tant que cause et le fondement d'une stratégie territoriale pour la RSE, les entreprises réalisent de la valeur ajoutée à leurs stratégies 
de commercialisation, les habillant dans des causes de valeur sociale ayant un effet à long terme.

\section{Conclusions}

Les données les plus récentes du système E-Bacchus, pour l'Union européenne, contiennent 1291 appellations d'origine pour la production de vin.

L'illusion est que chacun de ces domaines ainsi décrites ont un niveau similaire de développement des termes des besoins du public, de la partie commerciale et de la réputation. Naturellement, il est normal que la RSE ait un profil différent dans les diverses régions. Par exemple, en Bulgarie l'utilisation des ressources de la RSE à l'appui des musées et de galeries et de leurs activités sur la préservation du patrimoine culturel contribuerait à renforcer la stabilité du secteur vitivinicole et augmenterait la confiance des communautés locales et amènerait effectivement à la rédaction du nouveau look de zones en stagnation. Dans la pratique, cela signifierait une utilisation efficace, appropriée et socialement bénéfique des ressources de la RSE pour la construction de la nouvelle présentation de la région et le rétablissement de sa réputation de marché.

Les ressources de la RSE dans le secteur vitivinicole sont toujours utilisées de façon insuffisante et peu effective pour améliorer la viabilité des régions, en particulier dans les régions périphériques avec une insuffisante stabilité du marché. Leurs études et leur compilation dans une base de données pour élaborer une stratégie locale contribuerait à l'utilisation rapide des bonnes pratiques et l'activation d'une ressource d'entreprise latente. 